\title{
HOW WELL DO DRIVERS UNDERSTAND THEIR OWN HEADLIGHTS?
}

\author{
Johnell O. Brooks, Richard R. Goodenough, Richard A. Tyrrell, Chris Guirl, \\ Kristin Moore, Nathan Klein, Laura Davis \& Tina Kubala \\ Clemson University, Department of Psychology \\ Clemson, SC, United States \\ Email: jobrook@clemson.edu
}

\begin{abstract}
Summary: The current research represents an initial investigation of drivers' understanding of high beam and low beam headlight patterns in a nighttime driving environment. Fifty-four university students used a highlighter to indicate on a paper diagram of a roadway scene the portion of the scene that they felt their headlights would illuminate. Although the headlight patterns produced by participants varied more than expected, several consistent patterns emerged. Classification and evaluation of these drivers' responses revealed that many drivers misunderstand the area of the roadway that is illuminated by their headlights. These misunderstandings fall into fairly distinct patterns. The results indicate many drivers possess an incomplete understanding of the pattern of illumination provided by their headlights. These results are consistent with earlier evidence that many road users fail to appreciate the visibility challenges that exist at night.
\end{abstract}

\section{OBJECTIVES}

Several lines of research suggest that road users do not appreciate, or even understand, the visual challenges associated with driving at night. More than thirty years ago, Leibowitz \& Owens (1977) hypothesized that drivers are unaware of the "selective degradation" of focal visual functions (i.e., the ability to recognize objects and hazards at night) in part because ambient visual functions, such as visual guidance and steering, are preserved at luminances that are typical during night driving. This perspective implies that drivers do not fully compensate for the visual challenges they face at night because the visuomotor task that most would consider to be fundamental to safe driving - keeping the vehicle in the intended lane - seems easy, and drivers receive little feedback concerning the extent to which their ability to detect and identify objects in the roadway has been degraded. More recently, research using modern driving simulators has supported the selective degradation hypothesis (Owens \& Tyrrell, 1999; Brooks, Tyrrell \& Frank, 2005). Additional evidence that road users fail to appreciate visual challenges at night comes from research showing that pedestrians dramatically overestimate their own visibility at night (e.g., Tyrrell, Patton, \& Brooks, 2004; Tyrrell, Wood, \& Carberry, 2004). These overestimates are greatest when the visual challenge faced by the approaching driver is maximal (low beams, dark clothing). Here, pedestrians' failure to appreciate the visual challenges faced by drivers has a clear consequence - pedestrians who are mistakenly confident of their own visibility are more likely to behave in ways that put them at risk and to assume that approaching drivers will easily avoid them. 
To further investigate the extent to which typical road users understand the challenges - and the most typical "intervention" - involved in nighttime driving, the current research investigates drivers' understanding of the beam patterns that are projected by high- and low-beam headlights.

\section{METHOD}

54 university students participated in exchange for extra credit in a psychology course. Students were presented with an 8.5 " by 11 " sheet of paper with two illustrations of a road scene (from Olson \& Farber, 2003, p. 132) each approximately 7" (w) by 4.5" (h). Each participant was instructed as follows: "This is a picture of a road. This middle line $\left(0^{\circ}\right)$ is the center of your lane. Please draw a shape (with a highlighter) that covers the area where you think most of the light falls when you are using low beams." Data collectors were instructed to ensure the shape was enclosed. Participants were then asked to complete the same task for high beams. Data was discarded for two participants, one due to incomplete data and the other due to a computer error.

After data collection was complete, each diagram was scanned to create electronic documents for use on a tablet PC. Each participant's high beam and low beam patterns were traced into Inkscape, creating a scaled vector graphic (SVG) file with three layers: the original road scene diagram, a low beam pattern layer, and a high beam pattern layer. The Python scripting language was used to combine multiple participants' data into one figure. Specifically, a group of SVG image files for individual participants were parsed into layers which were then grouped by high or low beam drawings and overlaid on a new SVG with the original roadway scene layer. The result was a composite of participant headlight drawings. Each participant's layer was set at an opacity of .1 (10\% opaque) allowing for translucent headlight patterns in the overlays with darker areas signifying more overlap in participant beam patterns.

In the first round of data coding, seven coders rated each participant's high and low beam pattern as one of the following 12 shapes: Horizontal Oval, Horizontal Rectangle, Vertical Rectangle, Igloo, Square, Circle, Triangle Pointing Up, Triangle Pointing Down, Trapezoid Pointing Up, Trapezoid Pointing Down, No consistent rating, and Other. If the majority of the coders identified a pattern as the same shape then the pattern was classified as such. If there was no majority identification, the pattern was classified as having no consistent rating. Preliminary composites were then created for both high beam and low beam patterns for each group.

After examining these preliminary composites, several groups were combined due to similarity of the shapes and a need for a more manageable number of categories. The final groups selected were Horizontal Ovals or Rectangles; Igloo; Square, Circle, or Vertical Rectangle; Triangle or Trapezoid Pointing Up; and Triangle or Trapezoid Pointing Down. The four high beam patterns and six low beam patterns classified as "Other" as well as the patterns with no consistent rating were excluded from the final composite diagrams.

In addition to categorizing the shape of each beam pattern, the following descriptive measurements were calculated for each category: 


\section{Size of sample}

This includes the total number of participants categorized within a given shape group in addition to the percentage of the entire sample.

\section{Width}

In order to discuss the horizontal extent of a participant's pattern, we developed a measurement in which each "block" on the road scene diagram equals five units. While these units are clearly arbitrary, they are useful for the discussion of pattern sizes and distributions. Width is defined as the distance between the leftmost edge of a pattern to rightmost edge of a pattern. The range, mean, and standard deviation are reported.

\section{Height}

The same measurement system used to calculate width was used to describe the vertical extent of a participant's pattern. Height is defined as the distance between the bottommost edge of the pattern to the topmost edge of the pattern. Note: height measures only the height of the pattern in itself. If, for example, the pattern were an inch from the bottom of the drawing, that inch was not included in the height measurement. Any portion of the drawing extending beyond the image of the roadway was not included in the measurements. The range, mean, and standard deviation are reported.

\section{Left / right bias}

This category was used to determine if the patterns were evenly distributed across the driver's lane of travel or if the shape has a bias towards the left or right. Each pattern within a shape group was categorized as more left, more right, or equal. A pattern was categorized as "more left" or "more right" when the majority of the width measurement was located to the left or right of the center of the lane $\left(0^{0}\right)$. A pattern was categorized as "equal" when the shape was evenly distributed on the left and right sides. "Mean left" and "mean right" describe the average of the width units on the left and right side, respectively. Standard deviations are also provided.

\section{Distance}

The final data describe the farthest reach of the pattern. Since the height measurement does not always start from the bottom of the road scene, we used a distance measure to determine how far the light extends down the roadway. For the distance measure, we created categories based on the distance indicators provided on the illustration ( $<25^{\prime}, 25-50$ ', 51-100', 101-200', 201-400', 401-horizon, and above the horizon).

Please see Figure 1 for the overlay composites for both high and low beams and Table 1 for the corresponding descriptive measurements. 

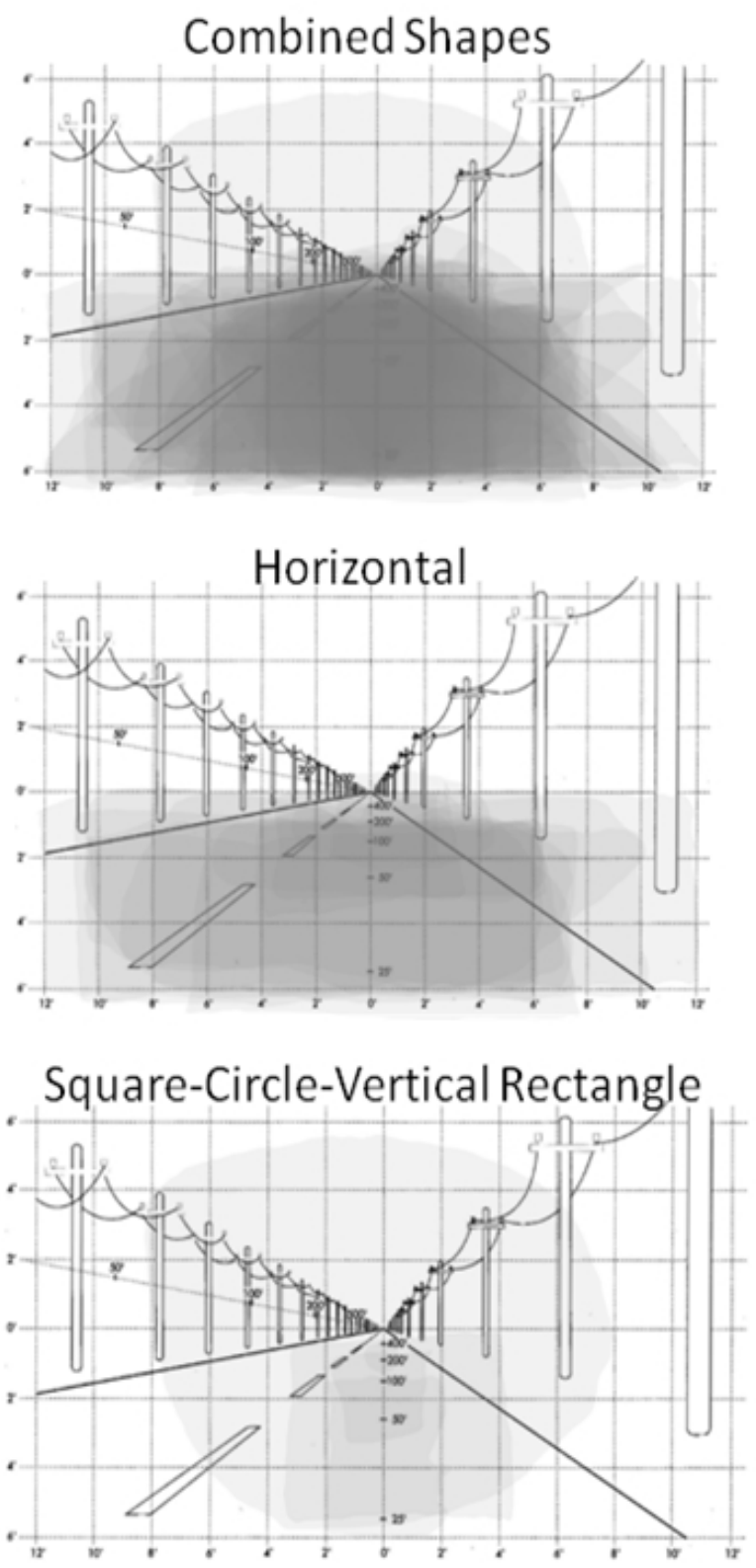

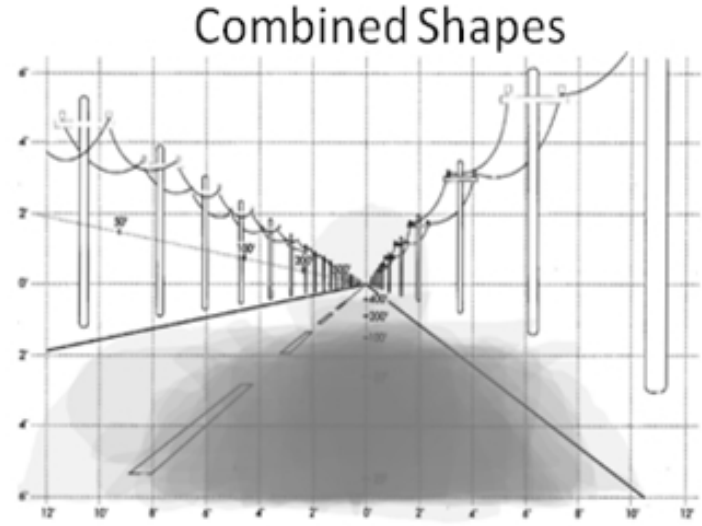

Horizontal

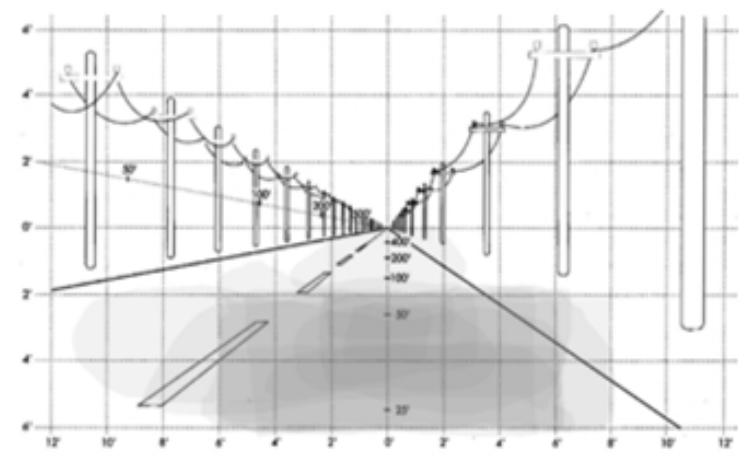

Square-Circle-Vertical Rectangle

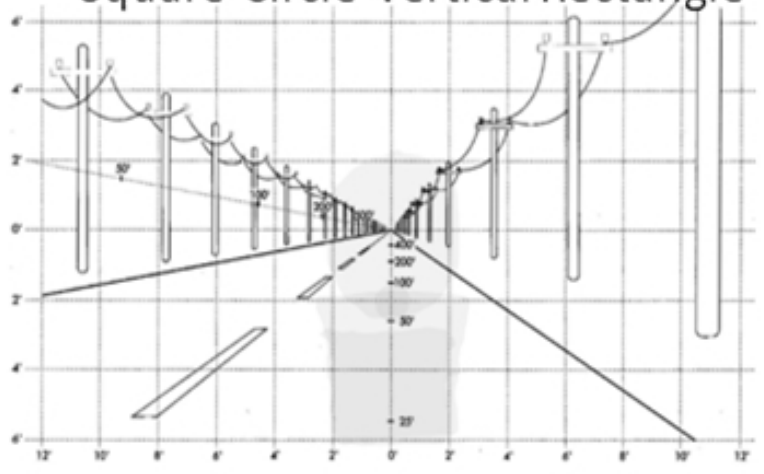

Figure 1. Overlay composites for high (left) and low (right) beams

\section{RESULTS \& CONCLUSIONS}

The purpose of this study was to quantify drivers' understanding of the illumination patterns that are projected by their high and low beam headlights. Using a simple highlighter-on-paper protocol, we were able to collect a reasonable amount of data in a short period of time. Transferring the data from paper to computer via a tablet PC allowed us to quantify, classify, merge, and display the data as composite diagrams. Upon final classification, $10.4 \%$ of high beam patterns fell into the Square, Circle, or Vertical Rectangle group while $29.2 \%$ fell into the Horizontal shape group. The remainder of high beam patterns were evenly distributed across the remaining three shape groups with about $20 \%$ falling into each. For the low beam patterns, $8.7 \%$ 

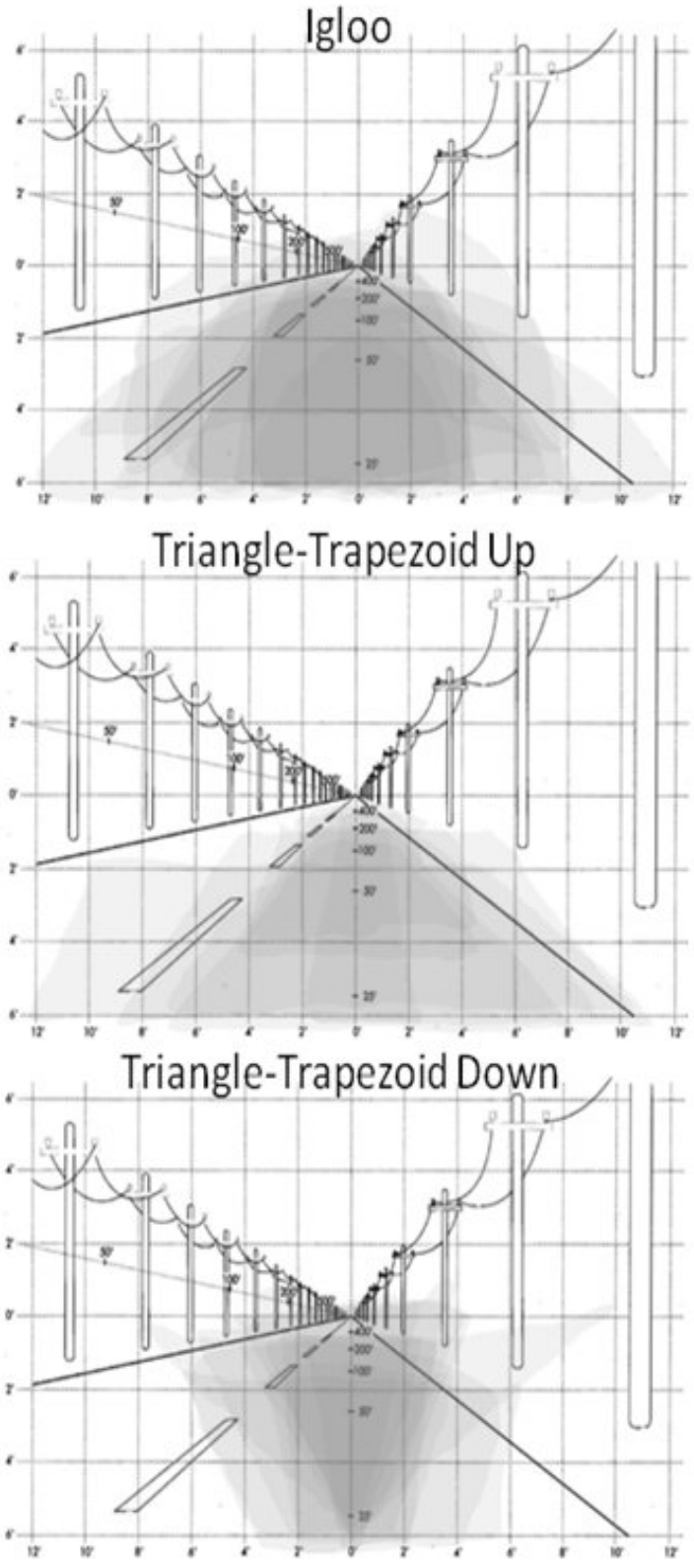
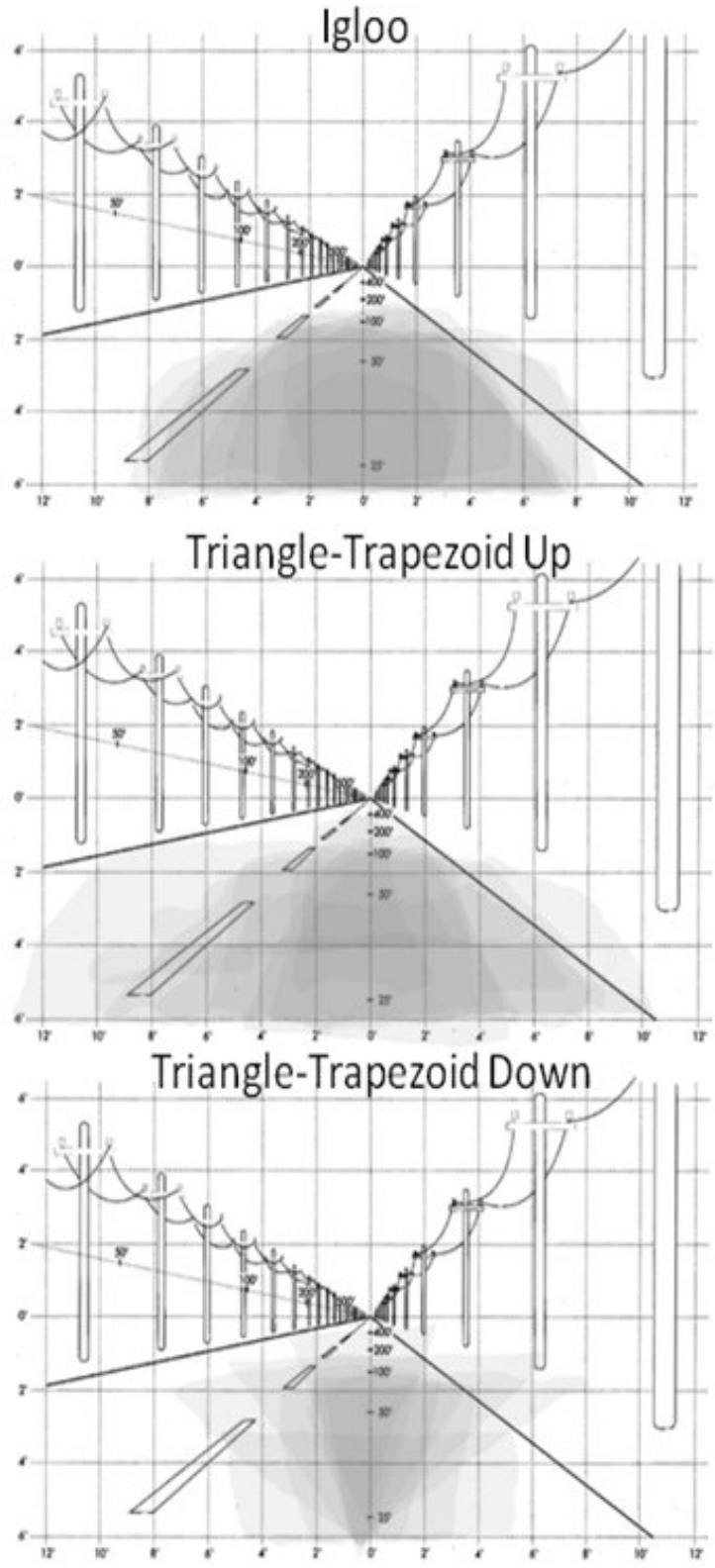

Figure 1 (continued). Overlay composites for high (left) and low (right) beams

fell into the Square, Circle, Vertical Rectangle shape group while $28 \%$ fell into the Horizontal shape-group. The remaining three shape groups comprised from $17.4 \%$ to $28.3 \%$ of the low beam patterns.

In order to quantify these beam patterns, we used a measurement system to allow for the discussion of the description of the drawings. The high beam patterns in the Horizontal and Igloo groups tended to have the broadest horizontal extent, while the low beam patterns belonging to the Igloo and Triangle or Trapezoid Up groups tended to have greatest horizontal extent compared to the other groups. Overall, the width mean and width range were generally consistent across groups, although the high beam values were a bit larger. When examining height, the 
Table 1. Descriptive measurements

\begin{tabular}{|c|c|c|c|c|c|c|}
\hline \multicolumn{7}{|c|}{ High Beam } \\
\hline & All Groups & $\begin{array}{c}\text { Square- } \\
\text { Circle- } \\
\text { Vertical } \\
\text { Rectangle }\end{array}$ & Horizontal & Igloo & $\begin{array}{c}\text { Triangle- } \\
\text { Trapezoid } \\
\text { Up }\end{array}$ & $\begin{array}{c}\text { Triangle- } \\
\text { Trapezoid } \\
\text { Down }\end{array}$ \\
\hline $\mathrm{N}=$ & 48 & 5 & 14 & 10 & 9 & 10 \\
\hline$\%$ of Group= & $100.0 \%$ & $10.4 \%$ & $29.2 \%$ & $20.8 \%$ & $18.8 \%$ & $20.8 \%$ \\
\hline \multicolumn{7}{|l|}{ Width } \\
\hline Range & 46.0 & 33.0 & 43.0 & 36.0 & 46.0 & 29.0 \\
\hline Mean & 25.7 & 14.2 & 30.6 & 30.7 & 24.3 & 20.7 \\
\hline Std. Dev. & 13.1 & 13.0 & 12.7 & 11.2 & 15.9 & 8.6 \\
\hline \multicolumn{7}{|l|}{ Height } \\
\hline Range & 24.5 & 24.0 & 9.0 & 8.0 & 12.0 & 5.0 \\
\hline Mean & 11.9 & 11.6 & 11.3 & 13.4 & 9.1 & 13.8 \\
\hline Std. Dev. & 4.2 & 9.1 & 3.3 & 2.2 & 4.3 & 1.7 \\
\hline \multicolumn{7}{|l|}{ Left/Right Bias } \\
\hline More Left & 18 & 1 & 5 & 6 & 1 & 5 \\
\hline More Right & 14 & 1 & 6 & 1 & 4 & 2 \\
\hline Equal & 16 & 3 & 3 & 3 & 4 & 3 \\
\hline Mean Left & 13.0 & 7.0 & 15.4 & 16.3 & 11.7 & 10.4 \\
\hline Left Std. Dev. & 7.0 & 6.6 & 7.5 & 6.1 & 7.8 & 3.9 \\
\hline Mean Right & 12.7 & 7.2 & 15.3 & 14.4 & 12.7 & 10.3 \\
\hline Right Std. Dev. & 6.4 & 6.4 & 5.6 & 5.6 & 8.2 & 4.9 \\
\hline \multicolumn{7}{|l|}{ Distance } \\
\hline$<25$ & 0 & 0 & 0 & 0 & 0 & 0 \\
\hline $25-50$ & 0 & 0 & 0 & 0 & 0 & 0 \\
\hline $51-100$ & 3 & 0 & 0 & 1 & 1 & 1 \\
\hline $101-200$ & 11 & 1 & 5 & 1 & 3 & 1 \\
\hline $201-400$ & 17 & 2 & 3 & 5 & 3 & 4 \\
\hline 401-horizon & 8 & 1 & 5 & 0 & 2 & 0 \\
\hline over horizon & 9 & 1 & 1 & 3 & 0 & 4 \\
\hline
\end{tabular}

\begin{tabular}{|c|c|c|c|c|c|c|}
\hline \multicolumn{7}{|c|}{ Low Beam } \\
\hline & All Groups & \begin{tabular}{|c} 
Square- \\
Circle- \\
Vertical \\
Rectangle
\end{tabular} & Horizontal & Igloo & $\begin{array}{c}\text { Triangle- } \\
\text { Trapezoid } \\
\text { Up }\end{array}$ & $\begin{array}{c}\text { Triangle- } \\
\text { Trapezoid } \\
\text { Down }\end{array}$ \\
\hline $\mathrm{N}=$ & 46 & 4 & 13 & 9 & 12 & 8 \\
\hline$\%$ of Group $=$ & $100.0 \%$ & $8.7 \%$ & $28.3 \%$ & $19.6 \%$ & $26.1 \%$ & $17.4 \%$ \\
\hline \multicolumn{7}{|l|}{ Width } \\
\hline Range & 45.0 & 10.0 & 30.0 & 22.0 & 39.0 & 26.0 \\
\hline Mean & 22.6 & 8.3 & 22.7 & 27.8 & 26.7 & 17.8 \\
\hline Std. Dev. & 11.4 & 4.5 & 10.0 & 8.2 & 13.6 & 9.3 \\
\hline \multicolumn{7}{|l|}{ Height } \\
\hline Range & 11.5 & 9.0 & 7.0 & 6.0 & 9.0 & 7.0 \\
\hline Mean & 9.2 & 8.0 & 8.1 & 10.9 & 8.1 & 11.3 \\
\hline Std. Dev. & 3.1 & 3.9 & 2.3 & 1.8 & 3.7 & 2.2 \\
\hline \multicolumn{7}{|l|}{ Left/Right Bias } \\
\hline More Left & 12 & 0 & 3 & 2 & 6 & 1 \\
\hline More Right & 16 & 1 & 5 & 3 & 3 & 4 \\
\hline Equal & 18 & 3 & 5 & 4 & 3 & 3 \\
\hline Mean Left & 11.5 & 4.0 & 11.5 & 13.9 & 14.0 & 8.6 \\
\hline Left Std. Dev. & 6.3 & 2.2 & 5.9 & 4.6 & 7.6 & 4.7 \\
\hline Mean Right & 11.2 & 4.3 & 11.2 & 13.9 & 12.7 & 9.1 \\
\hline Right Std. Dev. & 5.4 & 2.4 & 4.6 & 4.0 & 6.2 & 4.7 \\
\hline \multicolumn{7}{|l|}{ Distance } \\
\hline$<25$ & 0 & 0 & 0 & 0 & 0 & 0 \\
\hline $25-50$ & 9 & 1 & 2 & 2 & 3 & 1 \\
\hline $51-100$ & 23 & 2 & 10 & 4 & 4 & 3 \\
\hline 101-200 & 9 & 0 & 0 & 3 & 3 & 3 \\
\hline 201-400 & 3 & 0 & 0 & 0 & 2 & 1 \\
\hline 401-horizon & 1 & 0 & 1 & 0 & 0 & 0 \\
\hline over horizon & 1 & 1 & 0 & 0 & 0 & 0 \\
\hline
\end{tabular}

mean height for high beams was somewhat greater, although the overall range of heights for high beams was more than double the range for low beams. In order to determine if participants' perceive the distribution of light to be even across the roadway or more to the left or right of the lane, we examined whether the portion of each drawing falling on the left of the center line were greater than, less than, or equal to the portion to the right of the center line.

The data were relatively evenly spread between the three categories. This was fairly consistent with the categorical grouping of more left, more right, and equal as well as with the more objective measurements where the overall amount of the beam pattern was less than .5 unit different for both high and low beam. Finally, when looking at the distance of farthest reach of the beam pattern, a noticeable difference was observed between the high beam and low beam groups. For high beams, only three participants categorized the farthest reach as below 100 feet while 32 participants did so with the low beams.

\section{IMPLICATIONS \& FUTURE RESEARCH}

We have developed a quick and simple method of evaluating drivers' understanding of the patterns of illumination provided by their headlights. Classification and evaluation of these drivers' responses to this task revealed that many drivers misunderstand the area of the roadway that is illuminated by their headlights. These misunderstandings fall into fairly distinct patterns, with distinct patterns being repeated across participants. These patterns may become more 
distinct should participants consider not only the shape of the headlight beam, but also the distribution of light within it. Therefore, future research will ask drivers where in the beam pattern the highest amount of luminance is located, or if they feel light is spread evenly across the beam pattern. These more detailed responses would also provide a more accurate comparison of actual headlight beam patterns and distributions. Even though drivers are responsible to travel at a speed in which they can stop for hazards, if drivers do not understand how much light and the distribution of that light is provided by their head beams this may be an unrealistic task to ask of drivers (Leibowitz, Owens, \& Tyrrell, 1998).

\section{REFERENCES}

Leibowitz, H. W., \& Owens, D. A. (1977). Nighttime driving accidents and selective degradation. Science, 197, 422-423.

Leibowitz, H.W., Owens, D.A., \& Tyrrell, R.A. (1998). The assured clear distance ahead rule: Implications for nighttime traffic safety and the law. Accident Analysis and Prevention, 30, 93-99.

Olson, P.L., \& Farber, E.F. (2003). Forensic Aspects of Driver Perception and Response Second Edition. Lawyers \& Judges Publishing Company, Inc.

Tyrrell, R.A., Patton, C.W., \& Brooks, J.O. (2004). Educational interventions successfully reduce pedestrians' overestimates of their own nighttime visibility. Human Factors, 46, 170182.

Tyrrell, R.A., Wood, J.W., \& Carberry, T. (2004). On-road measures of pedestrians' estimates of their own nighttime conspicuity. Journal of Safety Research, 35, 483-490. 